\title{
The Viazis Classification of Malocclusion
}

\begin{abstract}
The scientifically unsubstantiated 120-year old classification of Edward Angle and its accompanying non-biologically based and arbitrary treatment goals no longer seem relevant or appropriate in modern clinical orthodontics. The author's review their years of work on identifying biologically based diagnostic terms centered on the clinical morphology of the alveolar bone and introduce a new biologically based and patient centered orthodontic classification. This classification accepts a patient's natural dentition within their own individual genetic morphologic appearance, rather than subjective or arbitrary "ideals", emphasizing treatment goals of improving alveolar bone morphology by maintaining stable occlusion.
\end{abstract}

Keywords: orthodontics, alveolar bone morphology, maxillary and mandibular hypoplasia, maxillary and mandibular hyperplasia, orthoeruption, orthodontosis, orthodontitis, orthomegaly
Volume 9 Issue 5 - 2018

\author{
Anthony D Viazis, ' Tom C Pagonis² \\ 'Orthodontist, Private Practice, Dallas, Texas, USA \\ ${ }^{2}$ Associate Clinical Professor, Tufts University School of Dental \\ Medicine; former faculty, Harvard School of Dental Medicine, \\ Boston, MA, USA
}

\begin{abstract}
Correspondence: Tom C Pagonis, Associate Clinical Professor, Tufts University School of Dental Medicine; former faculty, Harvard School of Dental Medicine, Boston, MA, USA, Email dental.research@comcast.net
\end{abstract}

Received: September 23, 2018 | Published: September 28 2018

\section{Introduction}

For nearly four years the authors have made the case and maintain that mainstream orthodontic treatment is based upon a flawed or even non-existent diagnostic process which leads to a flawed set of orthodontic classification terms. Moreover, there is an emerging and robust body of literature that continues to question and directly refutes the basis for conventional diagnostic classification of malocclusion. First introduced in 1899, Angle's classification of malocclusion in Class I ("ideal"/“normal"), II or III has remarkably endured and continues to be utilized as the main language of malocclusion. Angle's classification serves as the basis for orthodontic treatment planning for a large majority of orthodontic providers around the world, yet there is no evidence to suggest that this arbitrary "ideal" occlusion treatment goal provides significant health benefits or that it significantly improves oral function..$^{2-4}$ In 2002, Rinchuse \& Rinchuse stated that Angle's "normal" ("ideal)", is more accurately expressed as an extreme or a rare condition, affecting only 2 to $3 \%$ of the population. Furthermore, the authors of this 2002 study questioned the wisdom of forcing a stable, functional mandibular position to change in order to achieve a morphologic occlusion that conforms to an arbitrary ideal. ${ }^{5}$ In the Readers forum of a 2005 issue of the American Journal of Orthodontics and Dentofacial Orthopedics it was stated that it is a false belief that one should always endeavor to establish "normal" Class I occlusion-a concept, referred to as a century old fixation that has been arbitrarily determined. Clinical experience suggests that it is valid to finish treatment to an acceptable esthetic, stable, and functional occlusion. ${ }^{6}$

Of note, a substantial number of educators question the use of Angle's system as he defined it. The results of an email survey revealed that about half of the respondents were dissatisfied with the Angle molar classification system and do not use the system in an academic setting. Many participants commented that molar classification was limited and should be avoided. ${ }^{7}$ The special section, "100 years of orthodontic history," published in the December 2015 issue of the American Journal of Orthodontics and Dentofacial Orthopedics, delineates the important events in the past 100 years of orthodontics. ${ }^{8}$ The manuscript provides an important time-line and history of orthodontics but omits two critically important clinicianscientists, Kaare Reitan and Per Rygh who fundamentally contributed to the biology of tooth movement. The significance of this omission strongly suggests that orthodontics has been more technologically driven rather than biologically or scientifically.

Angle's classification is not based on a verifiable biologically based constant or on observed and documented pathologic processes but relies on dental intercuspation or static occlusion which only takes place between 15 to 30 minutes per day. The logical and arguably unfortunate consequences of relying on arbitrary ideals rather than verifiable biologic constants in the diagnostic and classification process leads to a misdirected "one size fits all" orthodontic treatment. The need for a biologically based diagnosis which follows into a logical classification system is clear and compelling.

In 2014 the authors introduced a disease-based or biologically verifiable model of orthodontic diagnosis ${ }^{10}$ based on alveolar bone clinical morphology rather than subjective arbitrary "ideals". The disease-based term Orthodontosis ${ }^{\mathrm{TM}}$ was introduced and defined as the non-inflammatory deficiency of the alveolar bone in the axial plane caused by the displaced root(s) of the tooth, typically palatally or lingually. This term becomes more significant as the authors now hypothesize that Orthodontosis ${ }^{\mathrm{TM}}$ is the underlying precursor to the clinical manifestation of a cleft or, as defined, a split or separation of bone in craniofacial development. The soft tissue consequence or resulting excess soft tissue and chronic inflammation leads to the diagnostic term of Orthodontitis ${ }^{\mathrm{TM}}$. These terms serve as the basis of the cause or illness and disease in orthodontics. In 2017 the authors created the first set of classification terms which capture our disease-based model and identify Alveolar Hypoplasia and Alveolar Hyperplasia in both the maxilla and mandible. ${ }^{11}$ The purpose of this paper is to translate our observations and diseased-based diagnosis into a new biologically based orthodontic classification system. We therefore introduce the The Viazis Classification of Malocclusion based on the alveolar bone morphology and propose it as the new system for orthodontic classification as the valid replacement of Angle's invalid thesis. 


\section{Subjects and methods}

\section{Etiology and prevalence of malocclusion}

Much has been written on the etiology and prevalence of malocclusion with the underlying observation that addressing the complex nature of etiology is multi factorial. Many contemporary attempts have been made to classify normal occlusion based upon heredity, trauma, physical agents, habits, diseases, malnutrition, genetic influences, environmental influences and developmental defects of unknown origin. When observed in children the prevalence of malocclusion exhibits a broad range of $39 \%$ to $93 \%$. This broad range reflects variations in registration of malocclusions, identifying varying degrees of malocclusions (i.e. "mild" to "severe"), age and variations among ethnic groups. ${ }^{11-18}$

\section{Proposed Viazis Classification of Malocclusion}

This classification system is based on the morphology of alveolar bone and can be divided into two main categories of bone discrepancy: Alveolar Bone Hypoplasia and Alveolar Bone Hyperplasia. Each of these categories is based on the underlying diagnosis of Orthodontosis ${ }^{\mathrm{TM}}{ }^{10}$ for Hypoplasia and the introduction of Orthomegaly ${ }^{\mathrm{TM}}$ as the diagnostic component of Hyperplasia. These classifications identify the pre-treatment morphologic status of alveolar bone with the ultimate orthodontic goal of creating a natural dental arch morphology (Figure 1) with proper and natural orientation of tooth roots and with a stable occlusion irrespective of an Angle molar classification. Both of these conditions demonstrate Orthodontitis ${ }^{\mathrm{TM}}$ which is the pathology expressed as gingivitis in the presence of malocclusion. Treatment of these two conditions also leads to treatment of the accompanying Orthodontitis ${ }^{\mathrm{TM}} .{ }^{10}$ The treatment of Alveolar Hypoplasia or Alveolar Hyperplasia results in the establishment of the natural dental arch similar or identical to what would have resulted from the perfect/natural eruption of straight teeth. The authors believe that this fundamental treatment goal is achieved through orthodontic mechanotherapy that is best described as the facilitation of natural eruption or Orthoeruption ${ }^{\mathrm{TM}} .{ }^{10}$ In the case of Alveolar Hypoplasia this process induces alveolar bone remodeling and development by moving roots toward their final naturally erupted position from the beginning of treatment. In the case of Alveolar Hyperplasia, (maxillary or mandibular) treatment follows a similar sequence of attaching brackets and wires to the teeth exhibiting clinical spacing and closing of these spaces with elastic power chains. The restoration of the alveolar bone toward the establishment of a patient specific natural dental arch also treats associated soft tissue pathology or Orthodontitis TM (i.e.,persistent gingivitis in the areas of malpositioned teeth primarily due to the unique bacterial flora consisting of Eikenella corrodens and Treponema denticola). ${ }^{10}$

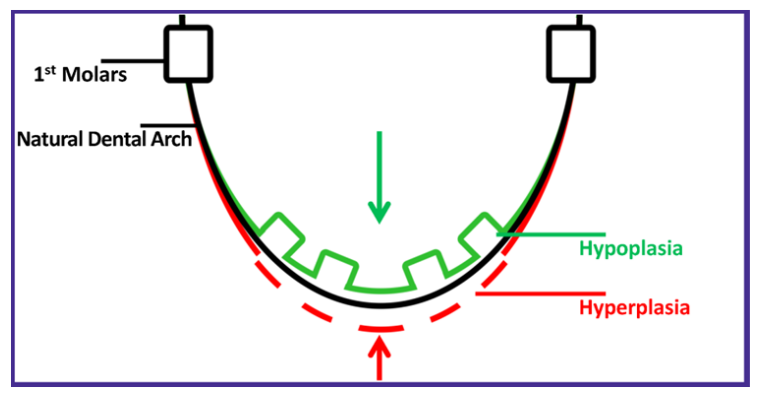

Figure I Graphic representation of alveolar bone morphology in hypoplasia and hyperplasia to a natural dental arch. Green boxes represent two lateral incisors and two premolars that are lingually displaced.
The pressure/tension theory of tooth movement is well accepted in orthodontics. The authors believe that in this model of tooth movement, orthodontic mechanotherapy that is gently administered along the entire root surface on the pressure side is clinically expressed in a painless remodeling of the osteocytes from osteoclastic activity. This is in stark contrast to tooth roots moving through the alveolar bone when heavier and arguably unnatural forces are used. On the tension side of tooth movement, the well accepted phenomenon of periodontal ligament (PDL) stretching takes place and is accompanied by increased blood flow resulting in the deposition of newly formed osteoid bone from osteoblasts. This newly formed osteoid bone is subsequently mineralized to the osteocytes of alveolar bone. The end result represents alveolar bone remodeling towards attaining a natural arch form. This is all possible of course because the unique osteocyte network of lacuna canalicular which allows for all these similar activities to take place and alter its mass and even remodel its structure in response to mechanical forces. An obvious example of the interdependence between the tooth root and alveolar bone is the mere fact that when the roots are removed from the mouth (i.e., the edentulous patient or a previous extraction site) the alveolar bone network disappears. This takes place because of the absence of nourishment from the PDL. The authors hypothesize that the presence of teeth or more specifically the PDL and associated tooth roots provides the potential for ongoing alveolar bone growth and remodeling provided that the orthodontic mechanotherapy is delivered properly.

The Viazis Classification of Malocclusion utilizes a straight forward and predictable two-step process which consists of the initial or step 1 identification of either Hypoplasia or Hyperplasia in respective dental arches followed by step 2 or the identification of categories and sub categories. These sub categories are specific both with regard to degree of Hypoplasia or Hyperplasia and account for the occlusal discrepancies of crossbite, underbite, overjet, openbite along with incisor proclination. In effect each sub category identifies the location and number of teeth exhibiting the referenced occlusal discrepancies and provides a specific treatment protocol for each and every condition. The main categories (step 1) and accompanying sub categories (step 2) are identified below and are numbered consistent with Table 1.

\section{Step I}

The clinician picks the Hypoplasia or Hyperplasia which applies as numbered consistent with Table 1 .

\section{Alveolar bone hypoplasia or orthodontosis ${ }^{\mathrm{TM}}$}

This clinical presentation typically exhibits the appearance of lingually malpositioned roots of teeth either in the maxilla or the mandible. This is accompanied by clinical deficiency or hypoplasia of the alveolar bone in the labial/buccal area of the affected teeth. The treatment goal is to orthoerupt malpositioned tooth root(s) towards their naturally erupted position thereby establishing natural alveolar bone architecture and form with a natural arch and with a stable occlusion (Figure 2).

\section{Alveolar bone hyperplasia or orthomegaly ${ }^{\mathrm{TM}}$}

The clinical presentation represents spacing of teeth due to excess alveolar bone albeit with normal architecture and normal intraboney orientation of all tooth roots either in the maxilla or the mandible. The treatment goal is to orthoerupt the malpositioned tooth roots thereby creating an alveolar bone architecture with no intra-arch or inter-arch spacing and an established of stable occlusion (Figure 3). 

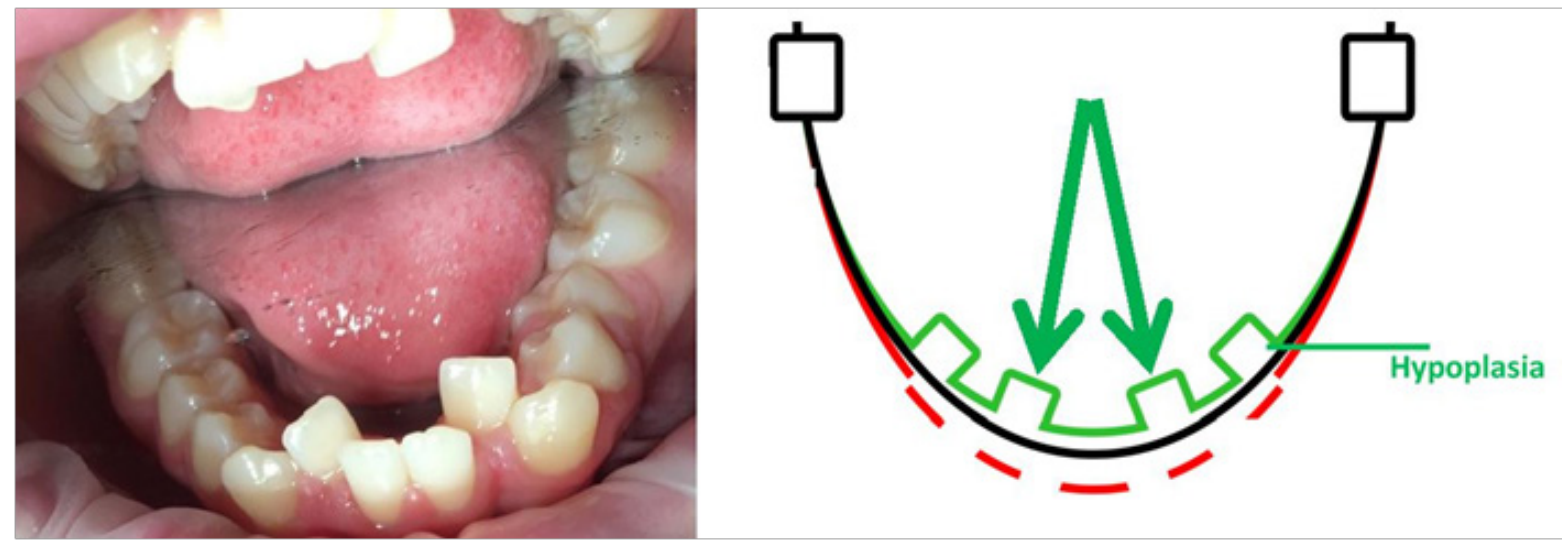

Figure 2A Before Pre-treatment clinical and graphic representation of hypoplasia or orthodontosis ${ }^{\mathrm{TM}}$.

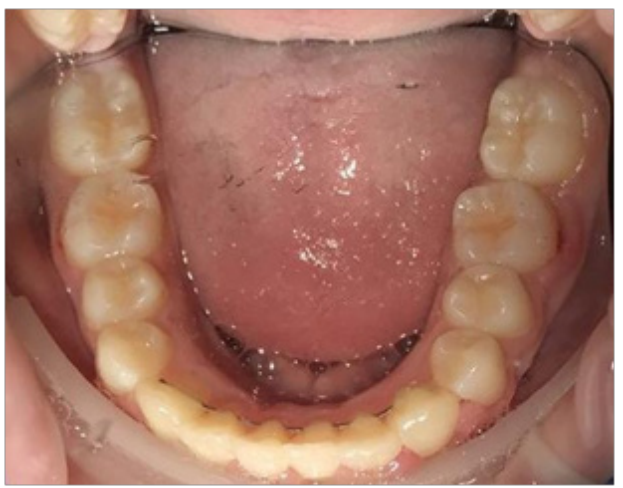

Figure 2B After Successful clinical post-treatment.
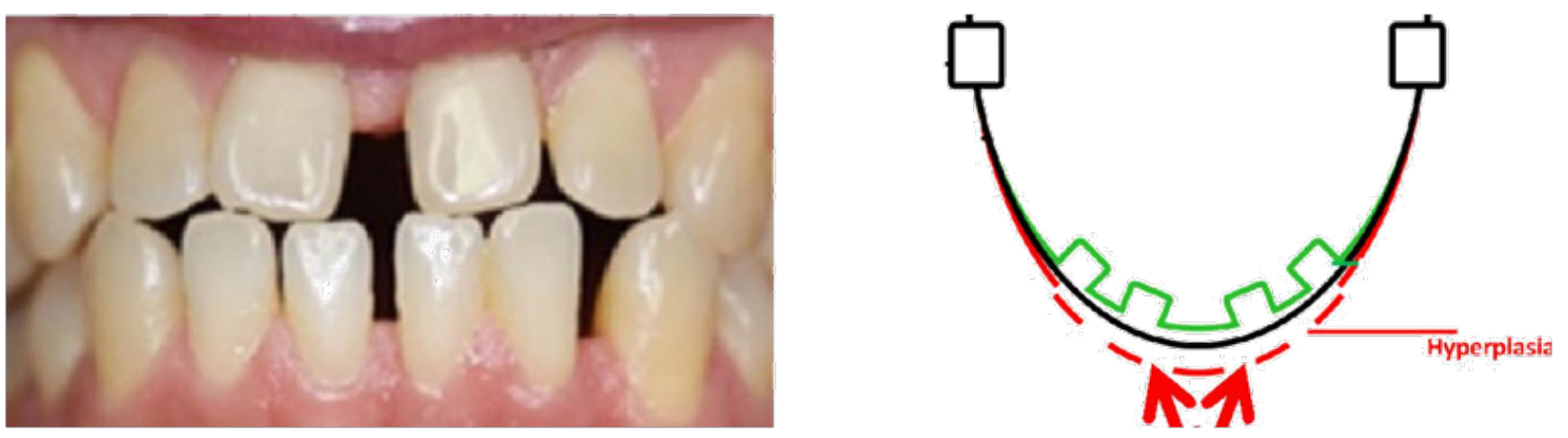

Figure 3A Before Pre-treatment clinical and graphic representation of hyperplasia or orthomegaly ${ }^{\mathrm{TM}}$.

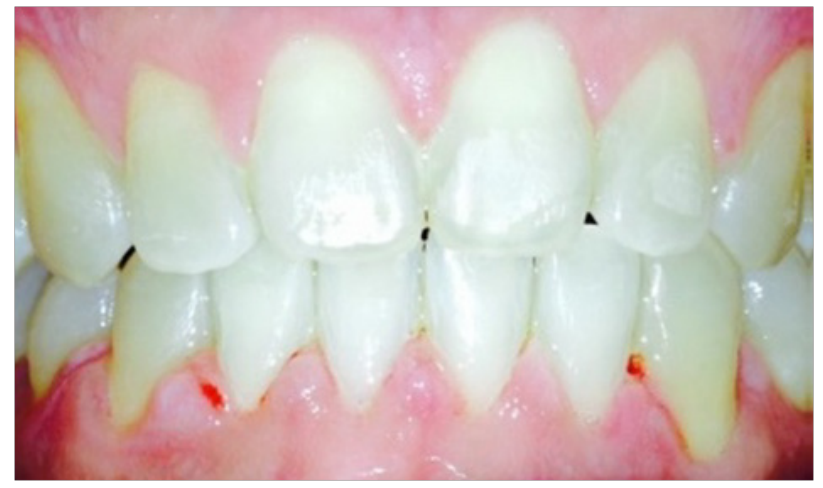

Figure 3B After Successful clinical post-treatment.

Citation: Viazis AD, Pagonis TC. The Viazis Classification of Malocclusion.J Dent Health Oral Disord Ther. 2018;9(5):363-37I. DOI: 10.15406/jdhodt.2018.09.00410 


\section{Step 2}

The clinician picks the category of Hypolasia or Hyperplasia which applies as numbered consistent with Table 1.

1. Anterior Hypoplasia

2. Generalized Hypoplasia

Or
1. Horizontal Hyperplasia

2. Vertical Hyperplasia

\section{Subcategories}

The clinician picks the sub category classification which applies. The sub categories are identified below and are numbered consistent with Table 1.

Table I The two step diagnostic checklist of the Viazis Classification of Malocclusion. The Palmer Orthodontic numbering system is used in the right column

\begin{tabular}{|c|c|c|c|}
\hline \multicolumn{4}{|c|}{ I. HYPOPLASIA } \\
\hline \multicolumn{4}{|c|}{ I.Anterior Hypoplasia } \\
\hline a. & Dental crossbite & One-two anterior teeth are in crossbite. & Lower 3-3 IPR \\
\hline b. & Dental underbite & Three or more anterior teeth are in crossbite. & Lower 6-6 IPR \\
\hline \multicolumn{4}{|c|}{ 2. Generalized Hypoplasia } \\
\hline a. & Minor & Has almost negligible hypoplasia. & Cosmetic dentistry \\
\hline b. & Moderate & The doctor can put the brackets exactly where they need to be on the teeth. & No IPR \\
\hline c. & Severe & $\begin{array}{l}\text { The doctor cannot put the bracket exactly where it } \\
\text { needs to be on the tooth (even if its only one) } \\
\text { because of its lingual position. }\end{array}$ & $\begin{array}{l}\text { Localized } \\
\text { IPR }\end{array}$ \\
\hline d. & Excess Dental Overjet & $\begin{array}{l}\text { In addition to the alveolar bone defect, the } \\
\text { overjet is more than } 3 \mathrm{~mm} \text {. }\end{array}$ & Upper IPR \\
\hline e. & Excess Dental Overbite & $\begin{array}{l}\text { In addition to the alveolar bone defect, the overbite } \\
\text { is such that no brackets can be placed on the } \\
\text { mandibular anterior teeth without bite blocks. }\end{array}$ & Biteblocks \\
\hline f. & Dental Openbite & $\begin{array}{l}\text { The dental arches are too small and the teeth are too big to } \\
\text { fit in the dental arch and procline forward. }\end{array}$ & $\begin{array}{l}\text { IPR of } \\
\text { big teeth }\end{array}$ \\
\hline \multicolumn{4}{|c|}{ 2. HYPERPLASIA } \\
\hline $\begin{array}{l}\text { I. H } \\
\text { (int }\end{array}$ & $\begin{array}{l}\text { lasia with horizontal spacing } \\
\text { h) }\end{array}$ & $\begin{array}{l}\text { The dental arche(s) demonstrate excess alveolar bone growth that is } \\
\text { expressed with spacing between the teeth. }\end{array}$ & Powerchains \\
\hline $\begin{array}{l}\text { 2. H } \\
\text { (int }\end{array}$ & $\begin{array}{l}\text { Dlasia with vertical spacing } \\
\text { h) }\end{array}$ & $\begin{array}{l}\text { The dental arches demonstrate excess alveolar bone growth that is } \\
\text { expressed with an opening between the dental arches. }\end{array}$ & 24/7 Elastics \\
\hline a. & Minor & Incisor proclination in addition to the opening & 3-3 IPR \\
\hline b. & Moderate & Incisor proclination in addition to the opening & 6-6 IPR \\
\hline c. & Severe & Incisor proclination in addition to the opening & 6-6 IPR (x2) \\
\hline
\end{tabular}

\section{Anterior hypoplasia with dental crossbite}

The condition where one or two anterior teeth are in crossbite (Figure 4). In addition to orthodontic mechanotherapy, this condition may require interproximal tooth reduction (IPR) from mandibular canine to canine.

\section{Anterior hypoplasia with dental underbite}

The condition where three to five anterior teeth are in crossbite (Figure 5). In addition to orthodontic mechanotherapy, this condition will require (IPR) from mandibular molar to molar. Six or more teeth in crossbite may indicate a skeletal underbite which may require orthognathic surgery.

\section{Minor generalized hypoplasia}

The condition where there is negligible hypoplasia of alveolar bone. The associated malalignment of teeth could possibly be resolved aesthetically with cosmetic dentistry alone (Figure 6).

\section{Moderate generalized hypoplasia}

The condition where the doctor can put the brackets exactly where they need to be on the teeth despite their lingual position (Figure 7). This condition may not require IPR.

\section{Severe generalized hypoplasia}

The conditions where the doctor cannot put the brackets exactly where there need to be on the teeth (even if it's a single tooth) because of their lingual position. This condition requires IPR on both the mesial and distal aspects of the bulky teeth (i.e. maxillary centrals, canines and premolars) in close proximity to those teeth where brackets are not ideally placed (Figure 8).

\section{Generalized hypoplasia with excess dental overjet}

The condition where, in addition to the alveolar bone defect, the overjet is more than $3 \mathrm{~mm}$ (Figure 9). This condition will require IPR of the maxillary arch. If the excess overjet is due to mandibular micrognathia then orthognathic surgery may be needed instead. 


\section{Generalized hypoplasia with excess dental overbite}

The condition where, in addition to the alveolar bone defect, the overbite is such that the clinician is unable to place brackets on the mandibular anterior teeth (or the maxillary teeth in the case of an underbite) without the use of bite blocks (Figure 10).

\section{Generalized hypoplasia with dental open bite}

The condition where the dental arches are too small due to the lingual position of the malaligned roots and accompanying clinical crowns are too large to fit in the respective dental arch. The large teeth appear proclined forward due to lack of available room for them to fit in the dental arch thus creating an open bite (Figure 11). With this accompanying open bite, IPR will be needed on all bulky sized teeth and possibly molar to molar in both arches.

\section{Hyperplasia with horizontal (intra-arch) spacing}

The condition where there is excessive alveolar bone growth in dental arches which is expressed by spacing between the teeth. The gradual power-chain force application from orthodontic mechanotherapy in these types of cases will remodel and restrict the alveolar bone to a smaller dental arch size thereby closing the dental spaces between teeth (Figure 12).

\section{Hyperplasia with vertical (inter-arch) spacing}

The condition where there is excessive alveolar bone of the dental arches but it is expressed by proclining of teeth forward rather than creating spaces within the dental arch. This is expressed with an opening between the dental arches. These cases require vertical elastics 24 hours per day. If the incisor teeth seem proclined after the opposing canines have come in contact with the use of the vertical elastics, IPR will be required on both the maxillary and mandibular arches to reduce this proclination and thus put the spacing into the dental arches and convert the vertical spacing to horizontal spacing. Adequate IPR will need to be performed until the maxillary lateral incisors overlap 1-2 mm over the corresponding mandibular incisors.

\section{Hyperplasia with vertical (inter-arch) spacing with minor incisor proclination}

The condition where there is minor proclination in addition to the opening (Figure 13A). This condition requires IPR canine to canine in both arches.

\section{Hyperplasia with vertical (inter-arch) spacing with moderate incisor proclination}

The condition where there is moderate proclination in addition to the opening (Figure 13B). This condition requires IPR molar to molar in both arches.

\section{Hyperplasia with vertical (inter-arch) spacing with severe incisor proclination}

The condition where there is severe proclination in addition to the opening (Figure 13C). This condition requires IPR molar to molar twice in both arches.
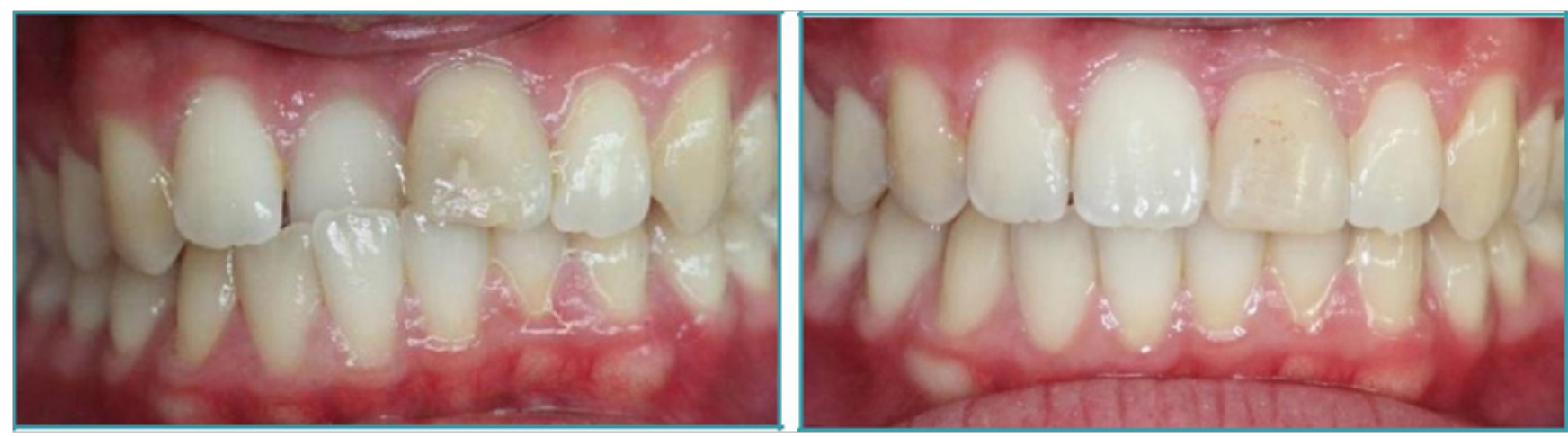

Figure 4 Before and after treatment of anterior hypoplasia with dental crossbite.
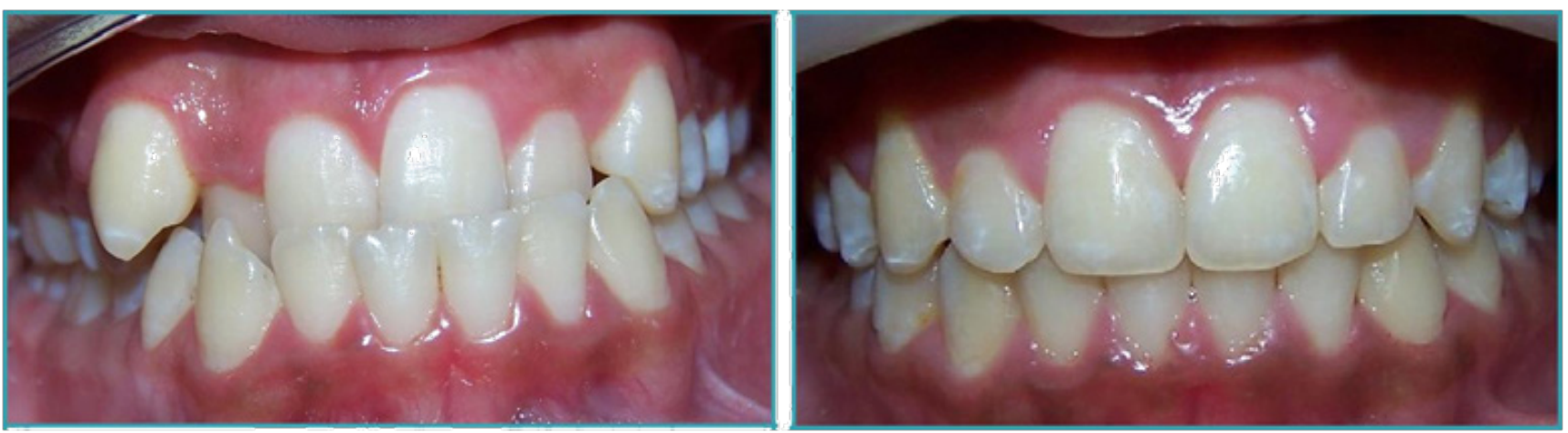

Figure 5 Before and after treatment of anterior hypoplasia with dental under bite. 

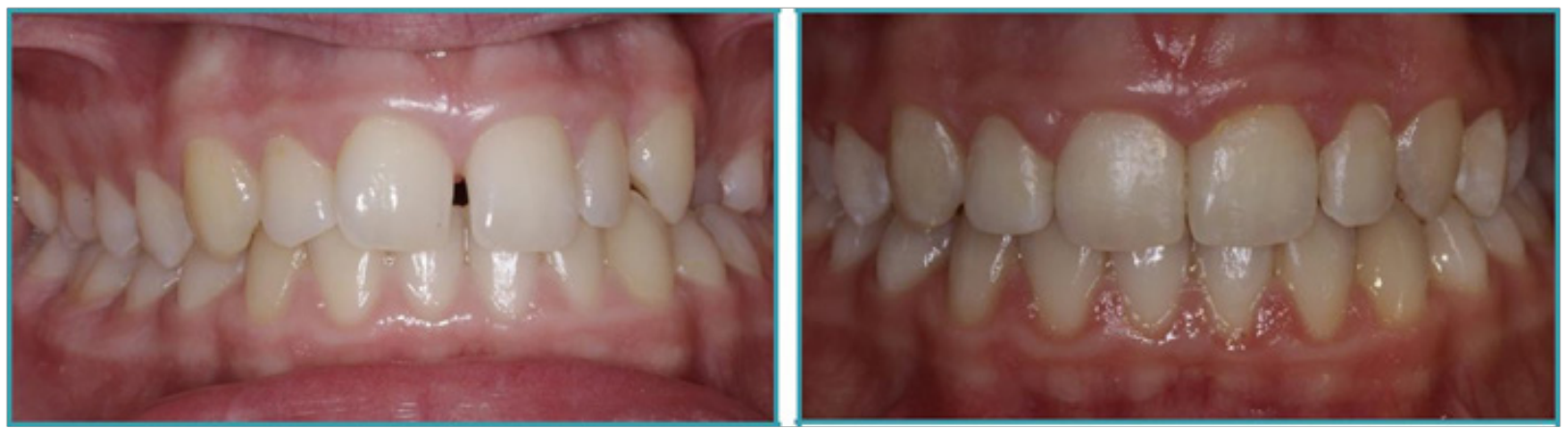

Figure 6 Before and after treatment of minor generalized hypoplasia.
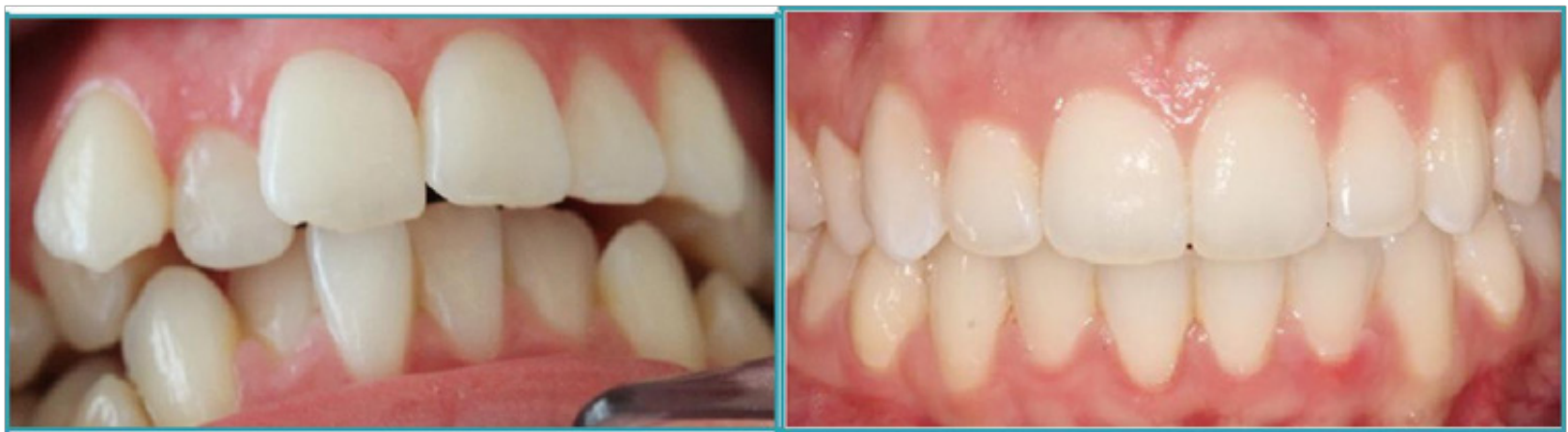

Figure 7 Before and after treatment of moderate anterior hypoplasia.

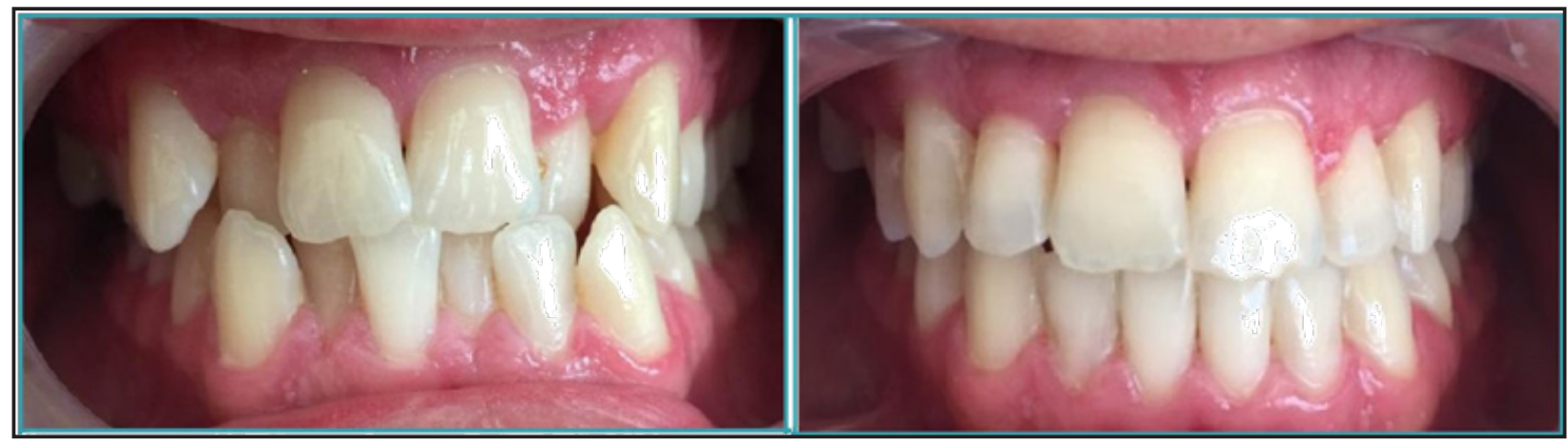

Figure 8 Before and after treatment of Severe Generalized Hypoplasia. Note in the before photo it is not possible to place a bracket correctly on the patient's mandibular left central incisor.
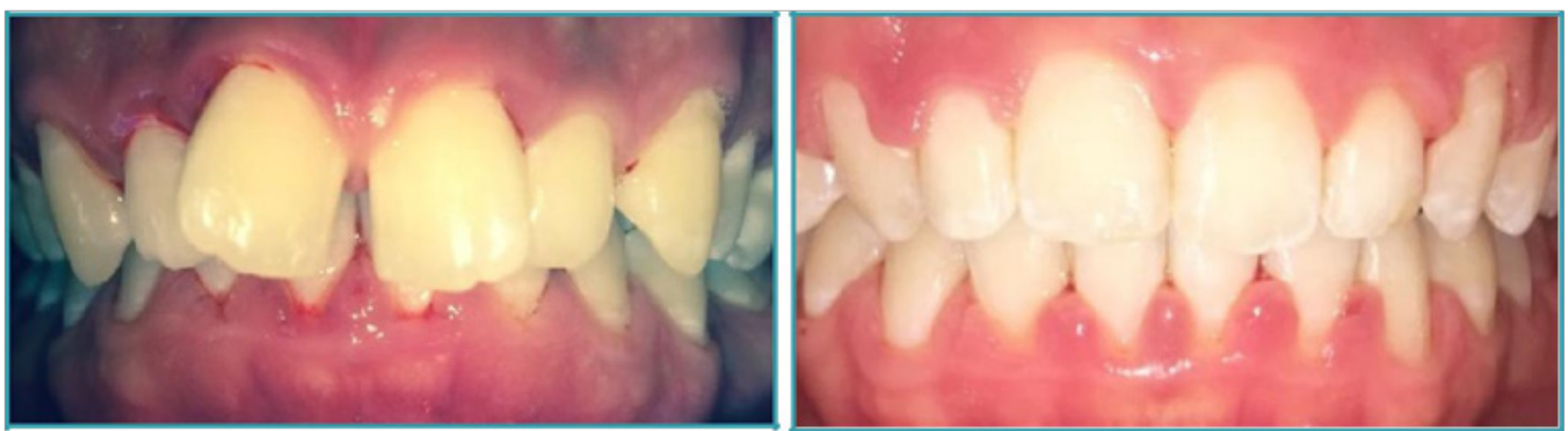

Figure 9 Before and after treatment of generalized hypoplasia with excess dental overjet. 

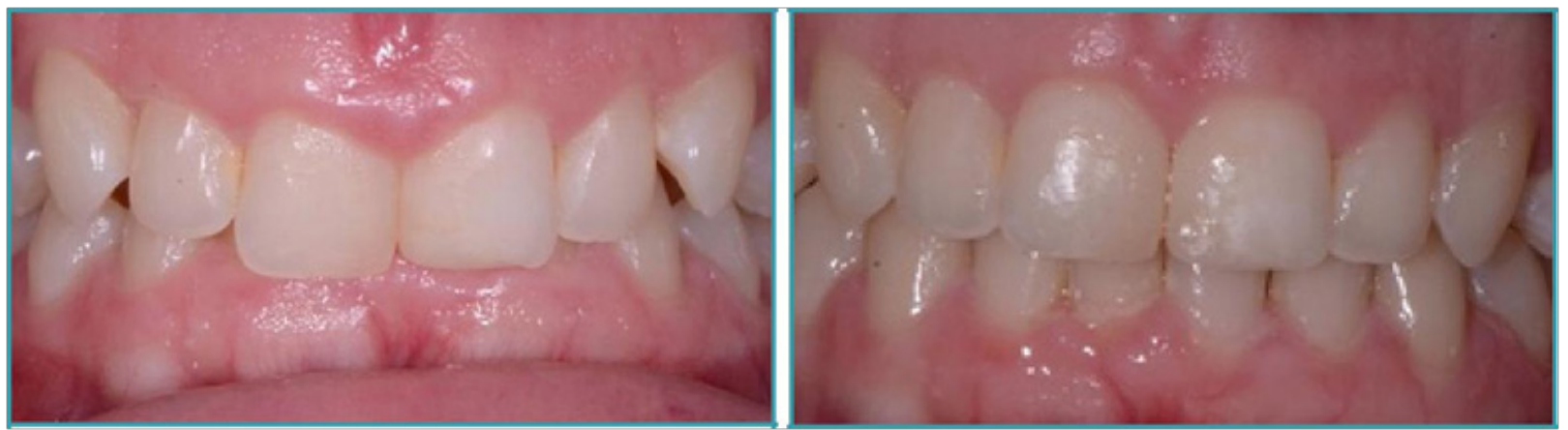

Figure 10 Before and after treatment of generalized hypoplasia with excess dental overbite.
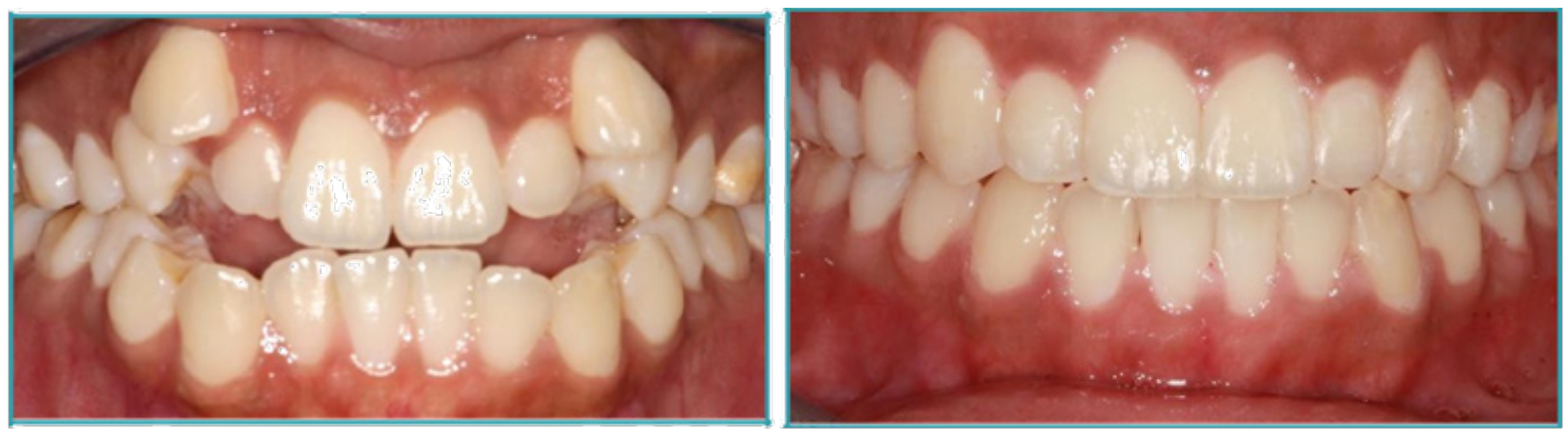

Figure II Before and after treatment of generalized hypoplasia with dental open bite.
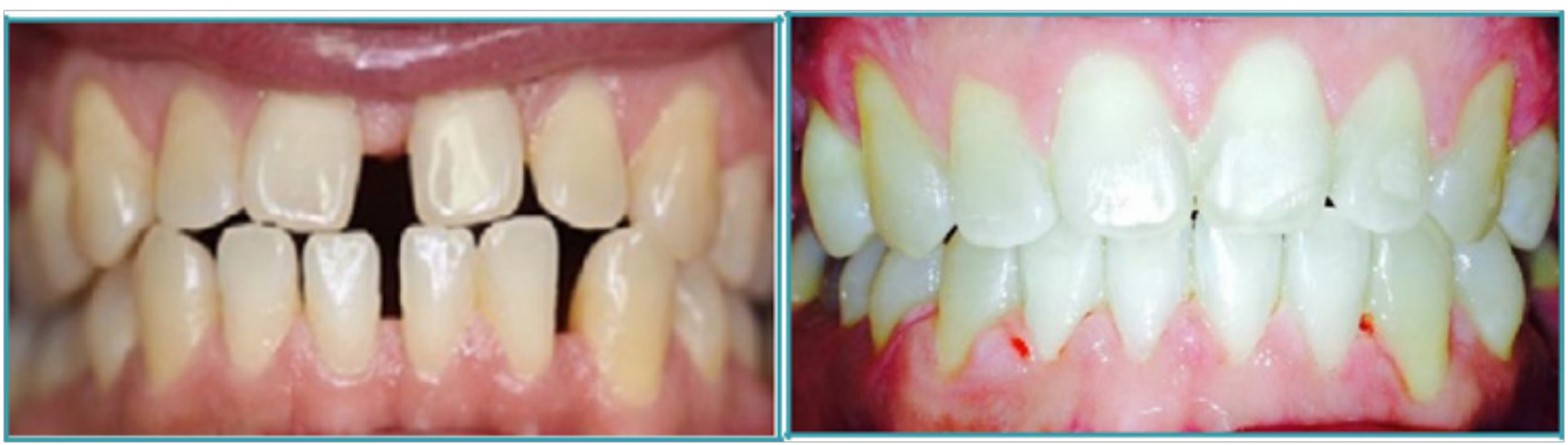

Figure 12 Before and after treatment of hyperplasia with horizontal (intra-arch) spacing.
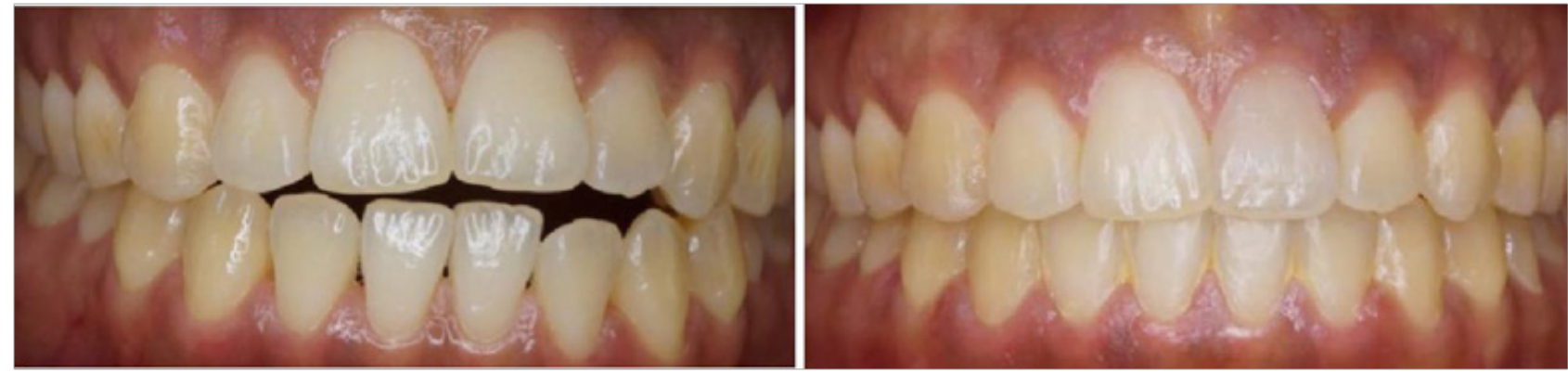

Figure I3A Before and after treatment of hyperplasia with vertical (intra-arch) spacing with minor incisor proclination. IPR performed from canine to canine. 

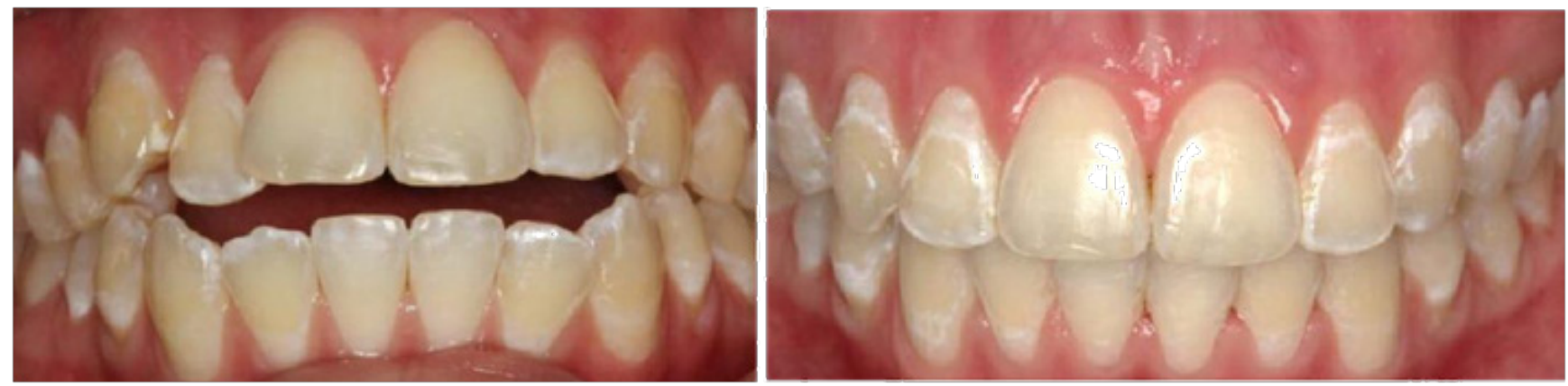

Figure I3B Before and after treatment of hyperplasia with vertical (intra-arch) spacing with moderate incisor proclination. IPR performed from first molar to first molar in both arches.
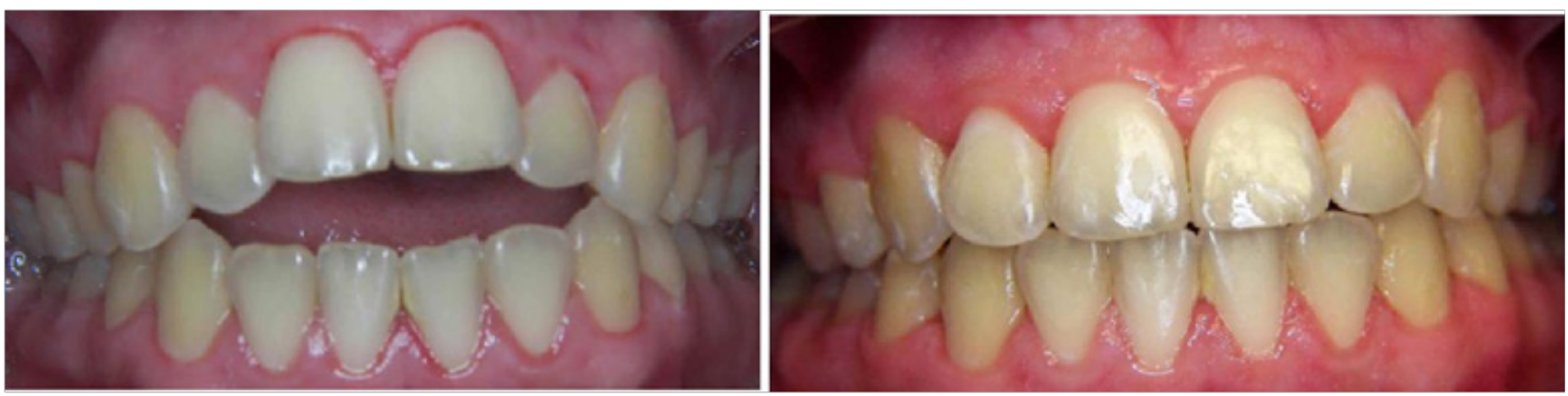

Figure I3C Before and after treatment of hyperplasia with vertical (intra-arch) spacing with severe incisor proclination. IPR should have been performed twice from first molar to first molar on both arches. However, IPR was done once resulting in the inadequate overbite of the left lateral incisor in contrast to the nice results demonstrated in Figures I3A and I3B.

\section{Discussion}

The authors believe the Viazis Classification of Malocclusion addresses a fundamental and large gap of knowledge towards the formulation of a biologically and disease-based orthodontic diagnosis that follows into a logical orthodontic classification system. This classification subsequently leads to patient centered treatment both non-extraction and non-surgically based upon the biological constant of alveolar bone morphology and the orthoeruption of tooth roots $^{10}$ rather than non-biological arbitrary "ideals". Several published clinical papers and case reports across a variety of clinical presentations identified and treatment planned with the Viazis Classification of Malocclusion have been successfully and predictably completed. ${ }^{10,19-23}$ The authors believe that every element of the Viazis Classification of Malocclusion embodies a biologically based etiology which leads to biologically based non extraction therapy without confusion. For example, the most common clinical expression of Alveolar Hyperplasia is the presence of spaces between the teeth in one or both of the dental arches. When this dental spacing occurs within a given dental arch and there is total intercuspation with the opposing arch or when dental arches exhibit parallel occlusal planes it is classified as Alveolar Hyperplasia with Horizontal Spacing. In other words, if one was to graphically connect all intra-arch spaces in unison during centric occlusion a horizontal plane parallel to the floor would be formed. The reason for the spacing is simply the development of excess alveolar bone. If one were to look at the development of excess alveolar bone on a continuum the extreme manifestation of excess alveolar bone development would cause both spacing and proclination of teeth especially in the anterior segment. If dental spacing with proclination of teeth occurs in both arches it presents as an anterior open bite or as Anterior Hyperplasia with Vertical (intra-arch) Spacing. The sub categories of Alveolar Hyperplasia with Vertical Spacing highlight the severity of the anterior open bite which is consistent with or proportional to the degree of proclination of anterior teeth. The general treatment recommendations for Alveolar Hyperplasia are logical and consist of power chains to close horizontal spacing and inter arch elastics to close vertical spacing. The degree of Alveolar Hyperplasia with Vertical Spacing is reflected in the sub categories or the degree of dental proclination. The treatment goal is to close the vertical spacing or convert it to horizontal spacing thereby creating a clear path to non- surgical, non-extraction treatment.

It is our belief that most or all clinical providers would accept the premise that the over-arching goal of orthodontic treatment is based upon improvement of function, dental and facial esthetics while maintaining or improving dental health. If this premise is accepted it is appropriate to have a solid foundation and understanding of etiology and disease which is biologically based and which leads to a logical classification system that supports the goals of treatment. It is important in orthodontics to re-examine and re-evaluate conventional "diagnosis" and classification; particularly its scope and meaning. A diagnosis should characterize the cause or nature of an illness and its criteria which can include signs and symptoms that represent the different features of disease in order to identify as many individuals as possible. A diagnosis and subsequent formulation of a classification should logically flow into each other because it represents the core or foundation of sound biologically based treatment. This is not the case with conventional orthodontic diagnosis and classification. Instead of a biological examination toward the formulation of an orthodontic disease and subsequent creation of a classification system, most clinicians remarkably adhere to Edward Angle's 1899 orthodontic 
thinking on occlusion by maintaining that the predictability and consistency of the maxillary first molar eruption pattern is of paramount importance. Furthermore, Angle stated that the fundamental scientific treatment goal of orthodontics is "the correction of the malocclusions of the teeth". This long enduring classification of malocclusion is neither diseased based nor a method for formulating treatment or assessing its prognosis. Simply stated it is a misdirection of treatment to merely address an observed occlusion towards an arbitrary "ideal". A sobering review of the literature just on the impact of using Class I, II and III on populations exhibiting a high and naturally occurring prevalence of bi -maxillary protrusion ${ }^{24-27}$ brings to light the shocking reality of an extraordinary number of teeth -perhaps in the billionsunnecessarily extracted to achieve an arbitrary "ideal".

Over the course of the last four years the authors have taken a thoughtful and methodical course towards redefining and bringing orthodontics into the mainstream of clinical dentistry by proposing reproducible and verifiable biologically based constants towards diagnosis and classification. We therefore propose the use of The Viazis Classification of Malocclusion.

\section{Conclusion}

For several years the authors, supported by a substantial body of literature have questioned the logic, foundation and accompanying orthodontic treatment philosophy of conventional orthodontic classifications. The truth is that Angle's 1899 classification of malocclusion is not biologically based and lacks the core biological or disease foundation to form a diagnosis - let alone a classification. The Viazis Classification is based upon the pre-treatment clinical morphology of the alveolar bone thereby utilizing the biologically based constant of alveolar bone rather than an arbitrary "ideal" occlusion which conforms to a molar relation derived from observation of static occlusion. Since the Viazis Classification of Malocclusion is based upon the biologically based morphology of the alveolar bone it logically follows into universal orthodontic treatment. This accepts a patient's natural morphologic appearance and stable occlusion irrespective of molar relationships and more importantly, irrespective of ethnic or racially attributed craniofacial / orthodontic characteristics. Simply stated an orthodontic classification and subsequent treatment should be universally applied regardless of facial profile towards an improved alveolar morphology by orthoerupting malpositioned tooth roots from the onset of treatment thereby inducing alveolar bone remodelling and development leading to non-extraction therapy. Emphasis needs to be placed on the true nature of malocclusion as a disease and not as a cosmetic alternative to fulfil an arbitrary and racially narrow-focused "ideal".

\section{Acknowledgment}

The authors wish to thank the Fastbraces ${ }^{\circledR}$ providers who supplied their case images.

\section{References}

1. Angle EH. Classification of malocclusion. Dental Cosmos. 1899;41:248 264.

2. Rinchuse D, Rinchuse D. Ambiguities of Angle's classification. Angle Orthod. 1989;59(4):295-298.

3. Gravely JF, Johnson DB. Angle's classification of malocclusion: an assessment of reliability. Br J Orthod. 1974;1(3):79-86.

4. Siegel NA. A matter of class: interpreting sub-division in a malocclusion. Am J Orthod Dentofac Orthop. 2002;122(6):582-586.

5. Rinchuse D, Rinchuse D. Orthodontics justified as a profession. Am J
Orthod Dentofac Orthop. 2002;121(1):93-96.

6. Prasad R. Class I occlusion a false goal? Am J Orthod Dentofac Orthop 2005;127(5):530.

7. Snyder R, Jerrold L. Black, white, or gray: Finding commonality on how orthodontists describe the areas between Angle's molar classifications. Am J Orthod Dentofac Orthop. 2007;132(3):302-306.

8. L Troehler. 100 years of orthodontic history. Am J Orthod Dentofac Orthop. 2015;148:875-876.

9. Khosravi R. One hundred years of orthodontic history. Am J Orthod Dentofac Orthop. 2016;149(4):445.

10. Viazis AD, Viazis E, Pagonis TC. The concept of a new dsental disease: orthodontosis and orthodontitis. J Dent Health Oral Disord Ther. 2014;(5):00030.

11. Viazis AD, ViazisE, Pagonis TC. Orthodontic Diagnosis Based upon Alveolar Bone Morphology. J Dent Health Oral Disord Ther. 2017;8(8):00316.

12. Andrews LF. The six keys to normal occlusion. Am J Orthod. 1972;62(3):296-309.

13. Roth RH. Functional occlusion for the orthodontist. $J$ Cli Orthod. 1981;15:32-51.

14. Ash MM, Ramfjord S. Occlusion. $4^{\text {th }}$ edition. Philadelphia: Saunders; 1996.

15. Rinchuse DJ, Kandasamy S, Sciotec J. A contemporary and evidencebased view of canine protected occlusion. Am J Orthod Dentofacial Orthop. 2007;132(1):90-102.

16. Moyers R, Riolo ML, Guire KE, et al. Differential diagnosis of Class II malocclusions: Part 1. Facial types associated with Class II malocclusion. Am J Orthod. 1980;78(5):477-494.

17. Graber TM. The "three M's": Muscles, malformation, and malocclusion. Am J Orthod. 1963;49(6):418-450.

18. Ackerman JL, Profitt WR. The characteristics of malocclusion: a modern approach to classification and diagnosis. Am J Orthod. 1969;56(5):443454.

19. Viazis AD, Viazis E, Pagonis TC. Non-Surgical Orthodontic Treatment of an Orthognathic Surgical Case. J Dent Health Oral Disord Ther 2016;4(5):00128

20. Viazis AD, Viazis E, Pagonis TC. Non-surgical Orthodontic Adult Molar Crossbite Correction and Sleep Apnea. J Dent Health Oral Disord Ther 2016;5(5):00168.

21. Viazis AD, Thaveeprungsiporn M, Pagonis TC. Alveolar Bone Remodeling and Development after Immediate Orthodontic Root Movement. J Dent Health Oral Disord Ther. 2017;6(2):00195.

22. Viazis AD, Viazis E, Pagonis TC. Alveolar Bone Growth with Orthoeruption. J Dent Health Oral Disord Ther. 2017;7(5):00258.

23. Viazis AD, Viazis E, Pagonis TC. The Biology of Orthodontic Treatment Time: Days Versus Years. J Dent Health Oral Disord Ther. 2017;8(1):00268.

24. Altemus LA. Frequency of the incidence of malocclusion in American Negro children age twelve to sixteen. Angle Orthod. 1959;29:189-200.

25. Fonseca RJ, Klein WD. A cephalometric evaluation of American Negro women. Am J Orthod. 1978;73(2):152-160.

26. Nummikoski P, Prihoda T, Langlais RP, et al. Dental and mandibulararch widths in three ethnic groups in Texas: a radiographic study. Oral Surg Oral Med Oral Pathol. 1988;65(5):609-617.

27. Reilly MT. Integumental profile changes after surgical orthodontic correction of bimaxillary dentoalveolar protrusion in black patients. $\mathrm{Am}$ J Orthod Dentofacial Orthop. 1989;96:242-248. 